\title{
Leaders
}

\section{Sport and exercise: the public health challenge}

John Dryden, in his poem, To my honoured kinsmen, writes: "The wise for a cure on exercise depend". This is good advice that the NHS is only just beginning to take seriously.

Current policy has rightly focused its attention on improving services to treat the big killers: coronary heart disease, stroke, and cancer. The challenge to reduce premature mortality from these diseases is huge-for example, the National Service Framework on Coronary Heart Disease, ${ }^{1}$ published earlier this year, has created a blueprint both for improving quality of services (from prevention to palliative care), and for improving access to these services across the country.

In the drive to modernise the NHS more emphasis is being placed on the important role that prevention and early intervention can play. Many are aware of the risk to health from smoking, but far fewer realise that the relative risk from physical inactivity is of a similar order. People who are physically inactive carry twice the risk of coronary heart disease and three times the risk of stroke as their more active counterparts. ${ }^{2}$

If it is to bring continued health benefits, physical activity needs to be embedded in the daily lives of many more people. Six out of ten men and seven out of ten women in England, between the ages of 16-74, are not physically active enough to benefit their health. ${ }^{3}$ The recently published National Diet and Nutrition Survey of 4-18 year olds has shown that children are becoming less active. ${ }^{4}$ About $40 \%$ of boys and $60 \%$ of girls spent, on average, less than an hour a day in activities of at least moderate intensity and therefore failed to meet our recommendation for young people's participation in physical activity. ${ }^{5}$

Moreover, participation in physical activity - such as, sports and walking, is strongly related to household income. However, this association between socioeconomic group and physical activity is partly offset by the higher levels of occupational activity in manual social classes. As a result, approximately $50 \%$ of men in social classes IV and V do not meet current recommendations in levels of physical activity, compared to two thirds of men in classes I and II.

Overall, the picture for women is worse. In social classes IV and V, nearly $80 \%$ of women fail to achieve the recommended level of physical activity. Although activity levels in class I are not significantly different, levels for social classes II and non-manual III are slightly higher.

Physical activity must be one of the most undervalued interventions to improve public health. Its benefits are great. Physical activity is closely associated with better health and reduced all cause mortality, including reduced mortality from coronary heart disease, stroke, colon cancer, and reduced fatality after a heart attack. Exercise helps to reduce blood pressure and hypertension, and can protect against the development of type II diabetes mellitus. It is a vital component of weight control. Weight bearing activity helps to maintain bone mass density and to reduce the risk of osteoporosis; and physical activity generally helps with improved balance, coordination, and endurance in older people. It has an important role in helping people to manage chronic conditions-such as, asthma and arthritis. It also has psychological benefits, including improved self esteem, and lower risk of mild to moderate depression. ${ }^{6}$ It is not surprising that exercise has been described by Professor Jerry Morris, one of the great figures in public health in the last century (and now in his nineties), as "the best buy in public health for the West".

Although the evidence of benefit, which has accumulated steadily over the past 10 years, is now compelling, some health professionals have not kept up with the developments in sport and exercise science. Few undergraduates receive any training in sport and exercise science during their medical training. It is small wonder that they feel ill equipped to give sound advice. Few exercise and sports science graduates are employed in the NHS. This is a challenge for health professionals. We need to be better equipped to meet the challenge of overcoming existing barriers to physical activity, both by simply extolling its virtues - telling people that it is good for them - and by helping with the practical provision of high quality, cost effective, and accessible programmes.

This is particularly important in addressing the socioeconomic gradient. There is evidence to show that the availability of accessible and affordable facilities is a major factor in the levels of physical activity among different groups. This is especially true for older adults.

But there are also wider influences: the fear of crime, which may deter some people from travelling; public transport strategies, which may deter people from walking or cycling; and the location of retail facilities, which may encourage rather than discourage car use. A policy to promote physical activity also needs to address factors such as these.

Primary health care professionals should see the promotion of physical activity as an integral part of their responsibilities. There have been several attempts to build these new responsibilities into a coherent and sustainable plan. Not all have been successful, ${ }^{8}$ but many are increasingly deserving of support. General practitioner referral schemes offer an opportunity to link public health and exercise, and to bring together the patient, the exercise professionals, and the health professionals in a partnership for better health.

I believe that the opportunities which exist in primary care for exercise promotion are gradually being recognised and exploited by the exercise science community. Some months ago, King called for primary care advice on physical activity to be given in conjunction with referral to appropriate community organisations, in order to facilitate long term increases in exercise participation rates. ${ }^{9}$ It is a view I share because it recognises the significance of multiagency collaborative working in public health. A good example of such joint working is a project to offer "exercise on prescription" in the Oldham area. This project was established in 1996 between Oldham Education and Leisure Services and West Pennine Health Authority, and Oldham Primary Health Care Teams, and is still in progress today. The scheme provides people with tailored exercise programmes to benefit their own particular health needs.

If we as health professionals must face a challenge, so must those involved in exercise and sports science. As with any health promotion activity, we need more convincing evidence about what interventions work and how to influence behaviour, particularly amongst those who are most at risk from their sedentary lifestyle. Sport and exercise 
medicine provides opportunities for working with people - to make them stronger, faster, more efficient, better able to deal with stress, and less susceptible to injury. This developing science covers physiological, psychological, biomechanical, and sociological influences on human performance in sport, work, and exercise. The evaluation of data collected from people engaged in sport, at work, or undergoing rehabilitation, supports the research base upon which that promotion is based. Rigorous evaluation of any intervention is vital if it is to be taken up by in modern public health programmes.

Chief Medical Officer, Department of Health,

79 Whitehall, London SW1A 2NS, United Kingdom

Liam.Donaldson@doh.gsi.gov.uk
1 Expert Group led by Prof George Alberti. Coronary Heart Disease: National Service Framezurk for Coronary Heart Disease : Modern Standards and Service Model. London: Department of Health, 2000.

2 Wannamethee G, Shaper AG. Physical activity and stroke in British middleaged men. BMF 1992;304:597-601.

3 Erens B, Primatesta P. Health Survey for England, 98. London: The Stationery Office, 1999

4 Gregory J, Lowe S, Bates CJ, et al. National Diet and Nutrition Survey: young people aged 4 to 18 years. London: The Stationery Office, 2000.

5 Riddoch C, Mutrie N, Parfitt G, et al. Young and Active? Young people and health enhancing physical activity - consensus statement. London: Health Education Authority, 1998

6 Physical Activity and Health: a report of the Surgeon General. Washington: US Department of Health and Human Services, 1996.

7 Morris JN. The role of exercise in the prevention of coronary heart disease: today's best buy in public health. Med Sci Sports Exerc 1994;26:807-13.

8 Reddish C, Puig-Ribera A, Cooper A. Effectiveness of physical activity promotion in primary care: a review. London: Health Education Authority, 1998

9 King A C. Role of exercise counselling in health promotion. Br f Sports Med 2000;34:80-1.

\section{Sports medicine training in the United States}

Medical training in the United States, particularly sports medicine training, professes some very interesting facets and practice opportunities. The specialty of sports medicine is a relative newcomer in the United States having only been conceived in the 1970s. Before its conception, most sports medicine was accomplished by orthopaedic surgeons and general practitioners. Because of the increasing demands of athletics in the United States and the need for doctors who can treat the "whole" athlete, including but not limited to the bones, tendons, ligaments, and muscles, the Primary Care Sports Medicine (PCSM) doctor has become increasingly popular at universities and with professional teams and elite athletes. The training of such doctors will be discussed with the hope of acquainting others with our practices and exchanging ideas with our international colleagues.

\section{Premedical education}

The education process in the United States starts with 13 years of publicly or privately funded study (basic education). Once a student attains a degree from this basic education (a high school diploma), they may apply to a college or university for undergraduate training. After acceptance in an undergraduate programme, the student must designate a major field of training. For the student interested in medical school, this has traditionally been biology, microbiology, chemistry, or one of the other sciences. A newer trend in the United States for those not interested in making a career in the basic sciences should they be denied a place at medical school is to designate a major in a career oriented field such as exercise physiology, microbiology, athletic training, physical therapy, or the newly forming physical therapy/athletic training (PT/ATC) programmes. The last of these is a combination of both athletic training and physical therapy, allowing the student more choices and higher salaries when choosing a job. Physical therapy has traditionally been a well paid job, but there is at present an overabundance of physical therapists in many regions of the United States. Having athletic training certification yields a superior candidate for these higher paying physical therapy positions yet still allows them access to athletes and sports teams.

\section{Medical education}

There are two camps of thought on medical training in the United States: allopathic and osteopathic medicine. Allopathic medicine is the more traditional route, employing conventional interventions for diagnosis and treatment. Its graduates are referred to as medical doctors (MDs). Osteopathic medicine is an alternative approach to medical training employing hands on manipulation of the body to assist in diagnosis and treatment of musculoskeletal and medical problems. They probably have better musculoskeletal training during medical school than their allopathic counterparts, but these differences may even out in residency. These physicians are referred to as osteopathic doctors (DOs). To apply for either of these medical schools, the candidate must have at least a four year undergraduate degree and scored high on the Medical College Admissions Test (MCAT). Typical scores are above the 80th percentile. Further criteria used to classify the large number of candidates for admission to medical school are grade point averages (individual grade point averages for science courses and courses required by the medical school are usually looked at separately), extracurricular activities, volunteer experiences (especially in the medical field), and any honours or awards. If the candidate meets the minimum acceptance criteria, they are given an interview with the faculty and staff of the medical school. At the interview, the candidate is asked questions about their desire to enter the medical field, motivations for career choices after medical school, and are assessed for their ability to communicate. Most medical schools assign numerical points to each area of the acceptance process and offer positions to the highest ranking candidates.

Once in medical school the student has specified curricula to attend. Many medical schools are adopting a problem based approach, which presents the student with a case through which they learn the areas of medicine (anatomy, physiology, pharmacology, social sciences, pathology, and others) and can apply it directly to a typical patient that they may encounter in practice. ${ }^{12}$ At most medical and osteopathic schools, the student is in the classroom the first two years with only limited clinical activities. The second two years are mostly clinically oriented in the setting of the hospital or office. Most schools allow elective rotations in those areas of interest for the medical student during this period. It is here that the 
student interested in sports medicine can become actively involved with the care of athletes. In addition to participating in an elective rotation in sports medicine, the student can also become involved by helping at sports physicals, observing sports medicine staff during sideline coverage, participating in research, etc. Rotations in orthopaedic surgery and/or primary care will also improve their chances of acceptance into a sports medicine programme. For an additional one to two years, some medical schools offer a Doctor of Medicine/Doctor of Philosophy (MD/PhD) or a Doctor of Osteopathy/Doctor of Philosophy ( $\mathrm{DO} / \mathrm{PhD}$ ) degree. This is highly attractive to students interested in research in the medical field and makes them ideal candidates for a sports medicine fellowship. On completion of medical or osteopathic school, students receive their medical doctorate (MD) or osteopathic doctorate (DO) and are considered a physician.

\section{Residency training}

Before being allowed to practice medicine independently as general practitioners, doctors must complete at least one year of training at a primary care residency programme (some states require a minimum of two years but this is extremely rare). Most doctors apply for and complete a residency in a designated field of medicine, either primary care or specialty training. The first year of their designated residency is termed the internship and the doctor is called an intern. Primary care residencies consist of paediatrics, internal medicine, emergency medicine, family practice, etc. Most primary care programmes are three years (internship plus two years). Many offer fellowships in areas of interests such as adolescent medicine, geriatrics, and sports medicine for an additional one to two years of training. Primary care has the advantage of a broad range of medical knowledge allowing the doctor to care for the whole patient. It has an extensive range of practice opportunities and carries the designation of primary care physician (PCP) which allows the doctor to act as a gatekeeper of medical utilisation among many insurance companies and federal agencies. Specialty residency training includes general surgery, orthopaedic surgery, ophthalmology, otolaryngology, pathology, radiology, anaesthesiology, etc. These are generally five year residencies (internship plus four years). Many of the specialty fields have subspecialties, such as cardiology, endocrinology, pulmonology, urology, vascular surgery, thoracic surgery, plastic surgery, etc, requiring another application process and an additional training period. These are the most specialised and highest paying fields in medicine in the United States. MD and DO residency programmes are sometimes separate, but there is often intermixing of doctors among these programmes.

\section{Sports medicine fellowships}

Several of the primary care specialties offer fellowships in sports medicine for an additional one to two years of training. There is an additional application process involved. Again, separate allopathic and osteopathic fellowships do exist but there is often intermingling of doctors among the programmes. Acceptance in a sports medicine fellowship is generally based on previous sports medicine experience, extracurricular activities, electives, and career desires. It is generally very beneficial if the applicant has done an elective rotation with the programme. Most programmes are accredited by a national board (American Board of Medical Specialties or the American Osteopathic Association) but overseen by its smaller member boards. ${ }^{3}$ Accreditation of a sports medicine programme requires the programme to have a minimum number of hours preserved to maintain the fellow's primary care training, a minimum number of hours of supervised sports medicine clinics with qualified personnel, have access to a broad range of specialists, as well as have exposure to numerous types of athletes. Specific learning objectives with respect to the athlete must also be met. These cover areas such as anatomy, physiology, biomechanics, pharmacology, nutrition, psychology, preventive care, conditioning and training, and management of injuries and illnesses. By graduating from an accredited programme, the resident is eligible for board certification in sports medicine (Certificate of Added Qualification) as overseen by its member board. Board certification is required at present for specialist reimbursement from most insurance companies ${ }^{4}$ and is favoured in teaching positions at accredited programmes. In the days of educated patients, board certified doctors will be actively sought out and those without certification will be rare (or very broke).

There are primary care programmes at present that are based in orthopaedic groups and are essentially designed to expand the groups sports coverage and reduce their office responsibilities by having a fellow for a very modest cost. Because they are not typically accredited programmes, these fellowships are ideal for the primary care resident whose career interests entail familiarity with sports injuries in their primary care practice, acquiring a position at an orthopaedic practice, or covering a few local sports teams.

There are also fellowships in sports medicine designed for the resident trained in orthopaedic surgery. They generally teach the surgical approach to sports medicine and do not stress the numerous other areas of athletic care. The fellows are not board eligible in sports medicine when their fellowship is complete. This means essentially that insurance companies reimburse them at the same rate for their surgeries as their colleagues without fellowship training. They do not have an accrediting body, which means there are no curriculum requirements or standards for supervision. Completion of a fellowship at a well known programme, however, can significantly improve a candidate's prospects at a desirable surgical sports medicine position allowing them to care for young healthy patients with few surgical complications and who are highly motivated to rehabilitate. It may rarely even involve team coverage as a head team doctor with a major sports team.

\section{Practice positions}

The PCSM fellow who is interested in maintaining qualifications in primary care and who would like to care for elite athletes will find job opportunities somewhat limited. Most job openings with this description are in academic fields, mostly residency programmes and fellowship programmes. A moderate number of positions are available in university student health clinics with promises of team coverage at the university and may include some sports medicine injury clinics. These are generally lower paying positions, have no call or inpatient requirements, and are not thought of by some to use the full training potential of the primary care doctor. There is an overabundance of positions for PCSM doctors in family practice settings, especially large groups, where their expertise is used to reduce the number of referrals to orthopaedic surgeons, curtail the number of unnecessary surgeries, and generate savings for the practice and insurance groups. The PCSM doctor may be able to charge as a specialist with some insurance companies under certain circumstances and still maintain their primary care status. ${ }^{4}$ Some orthopaedic surgery groups are very interested in hiring PCSM doctors to manage their non-surgical patients, perform presurgical clearances, expand team coverage, and sometimes to act as inpatient consultants. These are generally high paying jobs, but most do not use their primary care training enough to maintain their board certification and risk losing their PCP status. 
Table 1 Years of training for a sports medicine physician including a breakdown of the years in each area of training

\begin{tabular}{ll}
\hline Specialty choice & Years \\
\hline Primary care & \\
Basic education & 13 \\
Undergraduate degree & 4 \\
Medical school & 4 \\
Residency training & 3 \\
Fellowship training & $1-2$ \\
Total & $25-26$ \\
Orthopaedic surgery & 13 \\
Basic education & 4 \\
Undergraduate degree & 4 \\
Medical school & 5 \\
Residency training & $1-2$ \\
Fellowship training & $27-28$ \\
Total & \\
\hline
\end{tabular}

It is hoped that the preceding information will be instructional to those not familiar with our policies and practices in the United States and may help some students interested in sports medicine make educated and directed decisions. The road to becoming a doctor of sports medicine can be a long one (table 1), but for those interested in this field it can be a very fulfilling and lifelong career.

SCOTT H GRINDEL

Fellowship Director, Primary Care Sports Medicine Fellowship Program

Saint Vincent Health Center

Erie, Pennsylvania, USA

sgrindel@svhs.org

1 Kaufman A, Mennin S, Waterman R, et al. The New Mexico experiment: educational innovation and institutional change. Acad Med 1989;64:28594.

2 Neufeld VR, Woodward CA, MacLeod SM. The McMaster M.D. program: a case study of renewal in medical education. Acad Med 1989;64:423-32.

3 Lombardo JA, Wilkerson LA. Clinical sports medicine training and accreditation: the United States experience [editorial]. Clin f Sport Med 1996;6:76-7.

4 Henehan M, Jones R. Primary care sports medicine in the managed care environment: coping in today's culture. Physician and Sports Medicine 1997; 25:96-106.

\section{Insulin-like growth factor in muscle growth and its potential abuse by athletes}

Skeletal muscle is an inherently plastic tissue. There is evidence to suggest that muscles are constantly adapting both in quantity and quality to the changing functional demands imposed by the types and amounts of physical activity routinely performed. To date, the evidence suggests that, in adults, activity induced adaptations of skeletal muscle are orchestrated by local-that is, tissue level as opposed to systemic - mechanosensitive mechanisms, which appear to include a number of growth factors and hormones. Of particular recent interest is the growth hormone $(\mathrm{GH})$ /insulinlike growth factor-I (IGF-I) system. In the context of skeletal muscle homoeostasis, IGF-I is thought to mediate the majority of the growth promoting effects of circulating $\mathrm{GH}$. In addition, it appears to function in a $\mathrm{GH}$ independent autocrine/paracrine mode in this tissue. ${ }^{1}$

As information on the mechanisms that modulate muscle adaptation has been elucidated in the scientific literature, it is tempting for athletes to apply this knowledge to enhance muscle mass and hence function by artificially manipulating these systems. In some cases, this has led to simplistic notions that exogenous anabolic agents can be used to safely and effectively stimulate or augment muscle. Unfortunately, many of these attempts have been unsuccessful, and, in truth, they ignore our understanding of the integrated nature of physiological systems.

The circumstances that militate against this approach are severalfold. The first and most obvious problem with anabolic substances is that they are invariably non-specific. Agents that can stimulate muscle cells to hypertrophy will undoubtedly have effects on other cells and tissues as well - for example, the impact of growth hormone on prostatic hypertrophy. Secondly, just as the body is made up of tissues and organs that function as an integrated whole, so muscle is comprised of a number of different cell types which must also function in unison. For example, a treatment that stimulates muscle cells to hypertrophy must also recruit fibroblasts to strengthen the connective tissues that will transmit the force generated by the muscle cells, and must also act to enhance angiogenesis and mitochondrial function. In the absence of this coordination, one may develop larger (therefore "stronger") muscle cells, but the application of this enhanced contractile function would serve only to damage the structure of the muscle when the unenhanced connective tissue fails.

With regard to manipulating IGF-I either directly or through GH, a number of results from animal studies are instructive. Researchers have long sought ways to mitigate the atrophy inducing effects of unloading on skeletal muscle. An animal model used to study this effect involves "tail suspension" whereby rats are placed in cages with only their front feet touching any surface. This results in muscle atrophy which mimics that seen in humans following space flight. When GH or IGF-I has been supplied exogenously during tail suspension, the results have clearly indicated that the mass of the normally weight bearing muscles was in fact conserved. However, owing to the effects of these treatments on other tissues, the overall body weight of the rats had increased. It was as if the growth and development programme from an earlier developmental stage had been re-activated. However, there was one difference. When compared with their body weight changes, the muscles had actually "grown" less- that is, the normalised muscle mass was less in treated than untreated animals - the end result of course being that the growth factor treated rats would actually be less well adapted to normal ambulatory activity than the rats that received no treatment at all.

In humans, attempts to augment muscle mass using IGF-I have had less dramatic impacts. In studies designed to overcome the loss of muscle in the elderly, the overall impact of experimentally increasing circulating IGF-I levels has been negligible. ${ }^{9-11}$ For example, in one study the investigators managed to double the circulating IGF-I levels in elderly subjects but found no effect on the rate of protein synthesis in muscles; nor was there any augmentation of strength. ${ }^{11}$ In addition to this disappointing result, the supplementation of IGF-I in otherwise healthy - that is, GH normal - people is associated with (1) moderate to severe hypoglycaemia (it is after all insulin-like), ${ }^{6}$ (2) decreased growth hormone secretion, ${ }^{48}$ (3) a shift from lipid to carbohydrate oxidation for energy, ${ }^{8}$ and (4) a general disruption of the insulin/glucagon system. ${ }^{86}$ The issue of augmenting IGF-I is rendered even more complex 
because the biological activity of IGF-I in the body is now known to be substantially influenced by the family of IGF binding proteins. ${ }^{3}$ For example, recent work on the effects of hypoxia on rat growth suggests that it is, in fact, the impact of IGF binding protein-3 that is more closely related to overall growth than is IGF-I itself. ${ }^{7}$

There is also a more troubling aspect of IGF-I that has only recently begun to emerge. In addition to a direct anabolic effect on skeletal muscle-for example, the production of more protein - it has become clear that IGF-I is also capable of stimulating the proliferation and differentiation of muscle stem cells (satellite cells). In animal studies, there is evidence to suggest that this process is obligatory for muscle hypertrophy to proceed. However, this evidence that IGF-I is mitogenic should serve as a cautionary note to those who would use this agent to promote an anabolic state. There is increasing evidence to suggest that IGF-I signalling may also participate in cellular transformation. ${ }^{2}$ Specifically, elevated IGF-I levels have been linked to prostate, colorectal, and lung cancers. ${ }^{5}$

In the light of the large number of potentially negative impacts, ranging from disruption of the insulin system to cancer, it would seem that the exogenous augmentation of IGF-I does not represent a very attractive or effective method of increasing muscle mass or function. Clearly, the therapeutic use of these powerful growth factors awaits more focused research on the mechanisms through which these mediators actually influence growth in the context of the whole organism.

Department of Physiology and Biophysics

G R ADAMS

University of California-Irvine, CA 92717-4560, USA

1 Adams, G.R. The role of IGF-I in the regulation of skeletal muscle adaptation. Exerc Sport Sci Rev 1998;26:31-60.

Baserga, R. The IGF-I receptor in cancer research. Exp Cell Res 1999;253:1-6.

3 Baxter, RC. Insulin-like growth factor (IGF)-binding proteins: interactions with IGFs and intrinsic bioactivities. Am F Physiol 2000;278:E967-76.

4 Carroll PV, Umpleby M, Alexander EL, et al. Recombinant human insulinlike growth factor-I (rhIGF-I) therapy in adults with type 1 diabetes mellitus: effects on IGFs, IGF-binding proteins, glucose levels and insulin treatment. Clin Endocrinol 1998;49:739-46.

5 Grimberg A, Cohen P. Role of insulin-like growth factors and their binding proteins in growth control and carcinogenesis. F Cell Physiol 2000;183:1-9.

6 Jenkins PJ. Growth hormone and exercise. Clin Endocrinol 1999;50:683-9.

7 Moromisato DY, Moromisato MY, Brasel JA, et al. Effect of growth hormone therapy in mitigating hypoxia-induced and food restrictioninduced growth retardation in the newborn rat. Crit Care Med 1999;27:2234-8.

8 Sherwin RS, Borg WP, Boulware SD. Metabolic effects of insulin like growth factor-1 in normal humans. Horm Res 1994;41(suppl 2):97-102.

9 Taaffe DR, Jin IH, Vu TH, et al. Lack of effect of recombinant human growth hormone $(\mathrm{GH})$ on muscle morphology and $\mathrm{GH}$-insulin-like growth factor expression. F Clin Endocrinol Metab 1996;81:421-5.

10 Taaffe DR, Pruitt L, Reim J, et al. Effect of recombinant human growth hormone on the muscle strength response to resistance exercise in elderly men. f Clin Endocrinol Metab 1994;79:1361-6.

11 Yarasheski KE, Zachwieja JJ, Campbell JA, et al. Effect of growth hormone and resistance exercise on muscle growth and strength in older men. Am $\mathcal{F}$ Physiol 1995;268:E268-76.

\section{Transmission of cutaneous infections in athletes}

Myriad dermatoses can affect athletes. One of the most common cutaneous manifestations of athletic activity are skin infections. Bacteria, ${ }^{1-4}$ viruses, ${ }^{12}{ }^{4-8}$ and fungi ${ }^{12} 49-11$ cause these infections. Many are contagious and may have serious ramifications for team practices and competitions. Knowledge of these infections facilitates implementation of rapid treatment and preventive measures to ensure the least disruption in daily team activities.

Several specific sports related dermatological conditions are caused by bacterial infection. Staphylococcal infection is the most common but streptococcal infection also commonly occurs. ${ }^{1-4}$ Both organisms may present as varying clinical entities including impetigo, erysipelas, folliculitis, ${ }^{12} 4$ and furunculosis. ${ }^{3}$ In general, they are probably contagious to some degree. Impetigo, characterised by well defined, erythematous, yellow crusted, scaling plaques, and erysipelas, characterised by well defined, advancing, erythematous plaques, can be treated with topical warm soaks and oral antibiotics. ${ }^{14}$ Folliculitis presents as small follicular pustules that can be treated with topical or oral antibiotics. ${ }^{1}$ These bacterial infections occur in sports in which close personal contact occurs, including rugby, judo, and wrestling. ${ }^{24}$ Furunculosis outbreaks, however, have been noted also in football and basketball athletes. One study showed that $25 \%$ of high school athletes in these sports developed furunculosis. ${ }^{3}$ Direct contact with furuncles was significantly associated with transmission, while exposure to equipment seemed to be less important in its transmission. Some authors, however, have suggested that athletic bags and wrestling mats may also facilitate transmission of the organisms. ${ }^{4}$ Rapid treatment and isolation of the affected athlete from other competitors is of major importance in decreasing the rapid spread of the bacteria. ${ }^{12}$ Other authors have suggested that if the incidence of infection is low, then bandaging techniques may be a reasonable means to prevent transmis- sion. ${ }^{3}$ If outbreaks continue within a team, the bacterial carrier status of the members can be evaluated by culturing crural and nasal passages, ${ }^{4}$ and appropriate treatment can be instituted.

The three main viral infections that affect athletes are verruca (warts), molluscum contagiosum, and herpes simplex. Verruca can occur on any skin surface and can be transmitted by direct contact, but shared showers and locker room floors may also act as reservoirs. ${ }^{4}$ Swimmers may be particularly susceptible to plantar verruca. ${ }^{4}$ Destroying verruca will help to prevent it from growing and possibly causing pain. Furthermore the destruction may decrease transmission of the virus to other members of the team. Athletes with plantar verruca should wear sandals while showering in shared facilities. ${ }^{4}$ Molloscum contagiosum presents similar problems for athletes. This disease is characterised by discrete, white to skin coloured papules, and can be found particularly in wrestlers. Molloscum contagiosum can be, through direct contact, quite contagious and should be promptly treated with destructive methods to decrease the transmission to other athletes. ${ }^{14}$ Herpes simplex infection, known as herpes gladiatorum when identified in wrestlers, has been extensively reviewed. ${ }^{1246-8}$ Herpes simplex may also be endemic in rugby players. ${ }^{5}$ Clinically the infection can appear as an erythematous plaque upon which are vesicles. These vesicles may rupture resulting in erosions. In a study of one wrestling camp, $34 \%$ of participants were infected with herpes simplex. ${ }^{7}$ It is primarily transmitted through skin to skin contact, and transmission through fomites is felt to be less important. ${ }^{78}$ Identifying infected athletes promptly and excluding them from direct contact with other wrestlers will help to halt epidemic occurrences. ${ }^{78}$ Rapid administration of antiviral treatment may accelerate an athlete's return to wrestling. 
Fungal organisms can also significantly impact on athletes. Tinea pedis can be characterised by scaling plaques in a moccasin-like distribution on the plantar surfaces on the feet, by interdigital maceration, or by discrete vesicles on the foot. Tinea pedis can affect many athletes because the causative organism thrives in warm and moist environments. Runners, skaters, and long distance walkers may be particularly at risk. ${ }^{4}$ Affected athletes should be treated with antifungal agents and also wear protective footwear while using shared facilities to decrease transmission to other athletes. Athletes can also be infected with tinea corporis which is clinically characterised by well defined, scaling, erythematous, occasionally annular plaques. This infection has been extensively reported in wrestlers and has been termed tinea gladiatorum, trichophytosis gladiatorum, and tinea corporis gladiatorum. ${ }^{9-11}$ The term tinea corporis gladiatorum seems to be the most descriptive but is not comprehensive as it excludes tinea capitis which is transmitted between wrestlers. Several studies have examined the epidemiology of tinea corporis gladiatorum $^{6-11}$ in wrestlers. The frequency with which tinea corporis affects wrestling teams was found to range from $24 \%$ to $77 \%$ and varied with the methodology of each study. Two of these studies attempted to determine the incidence of tinea corporis in specific wrestling teams, both at the high school level. ${ }^{10}{ }^{11}$ One study, prompted by an alert from a local health department, noted an epidemic affecting $75 \%$ of a high school team's wrestlers ${ }^{10}$, and the other, investigating a team without a known epidemic, found $24 \%$ of the wrestlers infected with tinea corporis. ${ }^{11}$ Wrestling teams, often aware of the potential difficulties associated with tinea corporis infection, fastidiously clean equipment and mats. Transmission of the fungal organism, however, is most likely primarily through skin to skin exposure and not through fomites. ${ }^{10} 11$ This suggestion is supported by two findings. First, most lesions of tinea corporis gladiatorum are located on the head, neck, and arms, which are the areas of most contact between two competing wrestlers..$^{11}$ If wrestling mats played a larger role in transmission, one would expect more lesions on the lower extremities. Furthermore, attempts to culture organisms from mats have been unsuccessful. ${ }^{9}$ Athletes, particularly wrestlers and others subjected to extensive skin to skin contact, should be carefully examined before each practice and competition. ${ }^{10}{ }^{11}$ Athletes with signs of tinea infection should be treated promptly with antifungal agents and should be removed from direct contact with other wrestlers if the infected area cannot be appropriately covered using bandaging techniques.

Skin infections, to varying degrees, are transmitted among athletes. In general, data support the fact that most cutaneous infections are caused by close skin to skin contact. Prevention of skin infection epidemics in sports' teams is of paramount importance to avoid unnecessary morbidity and to minimise any disruption in team practices and competitions.

BRIAN B ADAMS

University of Cincinnati, College of Medicine, Department of Dermatology Cincinnati, OH 45267-0592, USA

adamsbb@email.uc.edu

1 Freeman MJ, Bergfeld WF. Skin diseases of football and wrestling participants. Cutis 1977;20:333-41.

Bergfeld WF, Taylor JS. Trauma, sports, and the skin. Am F Ind Med 1985; 8:403-13.

Sosin DM, Gunn RA, Ford WL, et al. An outbreak of furunculosis among high school athletes. Am $\mathcal{F}$ Sports Med 1989;17:828-32.

4 Powell FC. Sports dermatology. Fournal of the European Academy of Dermatology and Venereology 1994;3:1-15.

5 White WB, Grant-Kels JM. Transmission of herpes simplex virus type 1 infection in rugby players. $\mathcal{F} A M A 1984 ; 252: 533-5$.

6 Becker TM, Kodsi R, Bailey P, et al. Grappling with herpes: herpes gladiatorum. Am F Sports Med 1988;16:665-9.

7 Belongia EA, Goodman JL, Holland EJ, et al. An outbreak of herpes gladiatorum at a high-school wrestling camp. N Engl f Med 1991;325:90610.

8 Becker TM. Herpes gladiatorum: a growing problem in sports medicine. Cutis 1992;50:150-2.

9 Frisk A, Heilborn H, Melén B. Epidemic occurrence of trichophytosis among wrestlers. Acta Derm Venereol Suppl (Stockh) 1966;46:453-6.

10 Beller M, Gessner BD. An outbreak of tinea corporis gladiatorum on a high school wrestling team. F Am Acad Dermatol 1994;31:197-201.

11 Adams BB. Tinea corporis gladiatorum: a cross-sectional study. f Am Acad Dermatol 2000;in press. 